\title{
The pupil reflects motor preparation for saccades - even before the eye starts to move
}

\section{Stephanie Jainta*, Marine Vernet, Qing Yang and Zoi Kapoula}

IRIS Group, Physiopathology of Binocular Motor Control and Vision, CNRS UMR8194, University Paris V, European Hospital Georges Pompidou, Paris, France

Edited by:

Francisco Barcelo, University of Illes Balears, Spain

\section{Reviewed by:}

Stefan Pollmann, Otto-von-Guericke

University, Germany

Stuart R. Steinhauer, University of

Pittsburgh, USA

\section{*Correspondence:}

Stephanie Jainta, IRIS group,

Physiopathology of Binocular Motor

Control and Vision, CNRS UMR8194,

University Paris V, European Hospital

Georges Pompidou, Ophthalmology

Service, 20, rue Leblanc, 75908 Paris,

France.

e-mail: jainta@ifado.de
The eye produces saccadic eye movements whose reaction times are perhaps the shortest in humans. Saccade latencies reflect ongoing cortical processing and, generally, shorter latencies are supposed to reflect advanced motor preparation. The dilation of the eye's pupil is reported to reflect cortical processing as well. Eight participants made saccades in a gap and overlap paradigm (in pure and mixed blocks), which we used in order to produce a variety of different saccade latencies. Saccades and pupil size were measured with the EyeLink II. The pattern in pupil dilation resembled that of a gap effect: for gap blocks, pupil dilations were larger compared to overlap blocks; mixing gap and overlap trials reduced the pupil dilation for gap trials thereby inducing a switching cost. Furthermore, saccade latencies across all tasks predicted the magnitude of pupil dilations post hoc: the longer the saccade latency the smaller the pupil dilation before the eye actually began to move. In accordance with observations for manual responses, we conclude that pupil dilations prior to saccade execution reflect advanced motor preparations and therefore provide valid indicator qualities for ongoing cortical processes.

Keywords: pupil, saccade latency, gap paradigm, overlap paradigm

\section{INTRODUCTION}

The regulation of pupil size is a result of a complex interrelationship between the parasympathetic and sympathetic pathways of the human autonomic nervous system. Pupil constrictions or dilations mainly regulate the amount of light entering the eye (Lowenstein and Loewenstein, 1969). Besides this basic function, the observation of the dilation of the eye's pupil has a long history and well-described potential to help uncover cortical and subcortical processing activity (Hess and Howell, 1988; Beatty and Brennis, 2000; Wilhelm et al., 2002; Porter et al., 2007; Conway et al., 2008). Given that video-eye-tracking systems usually provide the user with additional information relative to pupil size, pupillometry might be of interest to a broad variety of research fields, especially those including clinical and human factor research areas. The present study is perhaps exemplary of how the additional data provided by pupillometry might further help to monitor ongoing cortical processing activity.

Recently, Hupe et al. (2009) demonstrated that pupil dilation begins as early as $700 \mathrm{~ms}$ before the time of a manual response. In so far as pupil responses are slow (Loewenfeld, 1999), the pupil responses were thought to be triggered by cortical processes which occurred prior to the manual reaction. These observations were in keeping with earlier reports by Richer et al. (1983) who showed that pupil responses reflect a slowly developing response preparation process in simple reactions (see also, Richer and Beatty, 1985). Furthermore, Karatekin et al. (2010) reported a comparison between a pro- and anti-saccade task; in an anti-saccade task participants are presented with a visual target on one side of a fixation dot but instructed to look away from it, i.e., they are instructed to make a saccade toward a blank location on the opposite side of the screen. This anti-saccade task produced longer latencies when compared to the pro-saccade task, and in parallel, absolute pupil diameters increased for anti-saccades. Additionally, the time after target onset at which the pupil diameter peaked increased in the anti-saccade task. We were therefore led to believe that if the pupil response could directly reflect motor preparations it might also appear within other standard paradigms involving saccade execution as well.

Saccades are generally a specific kind of motor response, since they show minimal conduction times and therefore reveal the shortest reactions times in humans. There is substantial knowledge about the different processes of motor preparation underlying different saccade latencies - from advanced automatic modes to completely controlled modes (see for example, Saslow, 1967; Findlay and Walker, 1999). Additionally, the use of different paradigms, like, for example, the gap and overlap tasks (or switching between the two of them), produces a corresponding variety of different saccades latencies, i.e., a variety of motor preparation. In the gap task, the fixation point switches off and a target appears after a gap period, while in the overlap task, the target appears while the fixation point is still on. Saccade latencies are typically shorter in the gap than in the overlap task and this gap effect is said to reflect an advanced temporal and spatial movement preparation during the gap period (Saslow, 1967; Fischer et al., 1993; Klein et al., 1995; Ross et al., 1996; Findlay and Walker, 1999). Basically, the extinction of the fixation point in the gap paradigm is thought to induce a warning signal, providing a state of readiness (temporal preparation) and also trigger the programming of the spatial components (spatial motor preparation). Some electrophysiological evidence for this argument showed that for monkeys the fixation-related neurons reduced their activity, while saccade-related neurons displayed 
phasic target-related responses during the gap period (Dorris et al., 1997).

Another way of inducing different motor preparations is the use of a switching task; generally, the switching design consists mainly in alternating between two different tasks in the same block of trials (mixed block) vs. performing each task in separate blocks of trials (pure block). Very recently, switching effects were reported for mixing pro-saccades triggered in gap and overlap trials, indicating that latencies for both tasks are longer when mixing trials relative to pure blocks; thus, alternating between automatic responses (gap) and more controlled saccadic responses (overlap) induced switching costs (Vernet et al., 2009). It should be noted that there is also substantial knowledge about the fixation system, which is active prior to saccades and which mainly includes the superior colliculus (Schiller et al., 1987; Munoz and Wurtz, 1992); the activity in this system has to be reduced in order to trigger saccades and especially express saccades, i.e., saccades with very short latencies, during gap paradigms. However, in order to avoid anticipation effects (see Materials and Methods), we did not include express saccades in our present study. We therefore concentrate on the classical use and interpretation of the gap and overlap paradigm.

To summarize, there is substantial evidence that the gap and overlap tasks - as well as switching between both - produce a variety of saccade latencies reflecting different motor preparations: the shorter the latencies, the more advanced the motor preparation relative to target onset. It was the aim of the present study to test whether different latencies produced by different saccadic tasks correspond to different pupil dilations. In other words, we sought to examine whether or not average pupil dilation would correlate with average saccade latencies produced by different saccadic tasks.

\section{MATERIALS AND METHODS PARTICIPANTS, APPARATUS, STIMULI, AND TASK}

We tested eight healthy and right handed participants (average age \pm SD: $37.4 \pm 12$ years; four males and four females) who had normal or corrected-to-normal vision. Each participant gave informed consent before the experiment and the research followed the tenets of the Declaration of Helsinki.

We used the gap and overlap paradigm (see Figure 1) in mixed and pure blocks to produce a variety of saccade latencies for each single participant. The set-up was comparable to Vernet et al. (2009).

The targets were presented binocularly on a computer screen as white dots $\left(0.2^{\circ}\right)$ on a black background (about $\left.10 \mathrm{~cd} / \mathrm{m}^{2}\right)$. In a constant, computer-controlled time protocol, the fixation dot was presented at the center of the screen for approximately $1,500 \mathrm{~ms}$ ( $\mathrm{SD}=100 \mathrm{~ms}$, in order to avoid predictability of the exact moment of the target onset; viewing distance: $57 \mathrm{~cm}$ ), while afterward the saccade target dot was presented for $1,500 \mathrm{~ms}$ either $\pm 7.5^{\circ}$ horizontally or $\pm 7.5^{\circ}$ vertically displaced. In the overlap conditions the fixation dot was present for $200 \mathrm{~ms}$ following the appearance of the target dot. For the gap conditions the fixation dot disappeared $200 \mathrm{~ms}$ before the target dot was displayed. The instruction was to look at the target as accurately and as rapidly as possible.

Pupil size and eye movement data were measured dynamically $(250 \mathrm{~Hz})$ using the EyeLink II; a chin and forehead rest was used
A GAP TRIALS

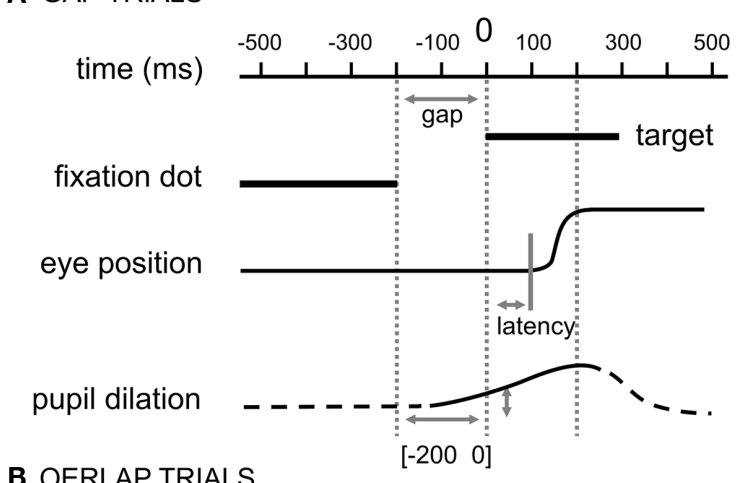

B OERLAP TRIALS

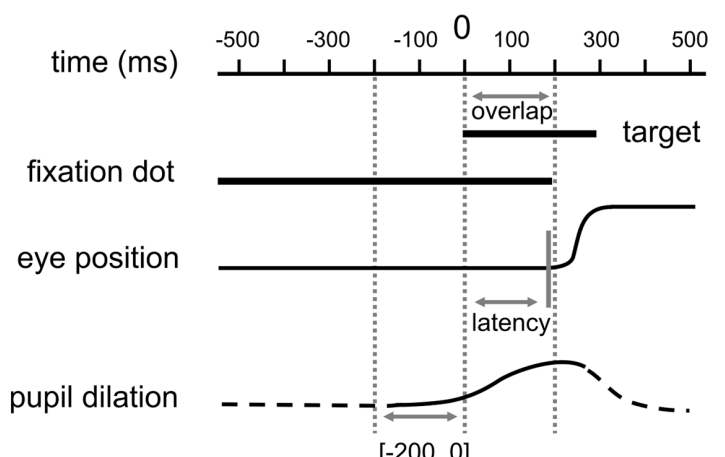

FIGURE 1 | Experimental paradigms. (A) Gap paradigm, the central fixation point disappeared $200 \mathrm{~ms}$ before the appearance of the eccentric target. (B) Overlap paradigm, the central fixation point remained illuminated for $200 \mathrm{~ms}$ after the appearance of the eccentric target. In both cases, the latency was the period between the onset of the target and the beginning of the saccade and the pupil dilation was calculated for the corresponding gap period [-200 0]. For illustration purpose, we included stereotypical eye movement and pupil response traces.

to fixate the head. The dark pupil system of the Eyelink II tracks the center of the pupil with a theoretical noise-limited resolution of $0.01^{\circ}$ (SR Research Ltd., Osgoode, ON, Canada). For the pupil size resolution the manual specifications of the Eyelink II system are $1 \%$ of pupil diameter.

During each session the participant performed four blocks: a pure gap block ( 60 trials), pure overlap blocks (60 trials) and two mixed blocks (64 trials) in which gap and overlap trials were interleaved. The order of the blocks was randomly assigned to each participant. Before each block a typical calibration sequence was run (see for example, Vernet et al., 2009).

It is important to note that one of the genuine characteristics of the gap and overlap paradigm represents a change in the visual presentation. In order to ensure that our reported data reflect changes in the motor preparations of the saccadic movements, some additional data concerning the baseline periods or single trial responses will be discussed.

\section{DATA PREPARATION}

A standard algorithm was used to detect saccade onset and offset in the calibrated eye position signals (Goldring and Fischer, 1997; Yang et al., 2002). Theoretical models suggest that saccades and 
express saccades (i.e., saccades with very short latencies) are produced by different sub-cortical circuits (Isa and Kobayashi, 2004; Schiller and Tehovnik, 2005); thus, we concentrated on saccades with latencies of 120-400 ms (i.e., excluding express saccades) in order to base our observations of pupil dilations on the same kind of saccades. Finally, and notwithstanding about $2 \%$ of anticipatory saccades, $19 \%$ of all saccades were categorized as express saccades and excluded from further analysis.

We analyzed the data for the pupil of the right eye and transformed the raw data from pixel units in $z$-scores for each participant. Unfortunately, we could not transform the raw data (pixel) into millimeter values since our set-up did not include calibrations of pupil dilations (we include some post hoc calculations in the Section "Appendix"). The mean across the interval [-300 -250] functioned as baseline; all trials were aligned at this mean baseline value, i.e., the actual baseline value was subtracted from all other observed values per trial (see Figure 1 for comparison). For each trial we calculated mean pupil sizes across $20 \mathrm{~ms}$ time windows before calculating the actual change before target appearance in order to reduce possible noise. Thus, the actual change of pupil size was calculated as (mean $[-1010]-$ mean $[-210-190])$. In other words, a pupil dilation was reflected in positive values while a pupil constriction was reflected in negative values. For the purposes of simplification, we will refer to the interval for which we calculated the pupil change as $[-2000]$ even though the actual change as (mean [-10 10] - mean [-210 -190]) would be the correct description.

Additionally, we calculated the pupil dilation for the period [-200 100] [i.e., (mean [90 110] - mean [-210 - 190])], in order to include a broader time period prior to the response which included the knowledge of the target position; strictly speaking, if one would doubt that there might already be some motor preparation prior to target appearance, this broader time period certainly contained motor preparation as the target location was known.

It is important to note, that even though we presented saccade targets for four different positions (up vs. down and left vs. right), we analyzed the pupil response only for a short period before the eyes started to move, i.e., while the participants were fixating centrally.

\section{STATISTICAL ANALYSIS}

In order to account for changes in pupil dilation and saccade latency, we used a linear mixed-effects model [lmer from package lme4 (Pinheiro and Bates, 2000; Venables and Smith, 2001) in R (www.r-project.org; 2008)]. Generally, linear mixed-effects models are based on maximum likelihood methods and commonly used in many areas (Baayen et al., 2008). The statistical package $\mathrm{R}$ provides reliable algorithms for mixed-effect parameter estimations as well as tools for their evaluation (West et al., 2007): the $p$-values and confidence intervals were estimated by using posterior distributions for the model parameters obtained by Markov Chain Monte Carlo sampling, typically including a sample size of 10,000 (see for example, Baayen et al., 2008).

In our present analysis we used saccade latency and pupil dilation as dependent variables in separate model estimations and included the tasks as factors of the independent variable into the regression analysis. As stated above, mixed-effects models explain data as effects on the first and second order statistics with respect to covariates and grouping structures. The covariate in our analysis was the task, and the grouping factor represented the participants. We will state the estimated coefficient with its SE, the $t$-value and the $p$-value for each effect of repetition; further, for the main results we will provide a table including all model parameters as well as estimated confidence intervals for the fixed effects of tasks.

\section{RESULTS}

\section{SACCADE LATENCIES AND AMPLITUDES}

Average $( \pm \mathrm{SD})$ saccade amplitudes were $6.7^{\circ}( \pm 0.4)$ for pure gap and $6.9^{\circ}( \pm 0.5)$ for pure overlap blocks. Saccade amplitudes for gap trials $(6.7 \pm 0.5)$ and for overlap trials $(6.6 \pm 0.4)$ in mixed blocks did not differ from those for pure gap or pure overlap blocks - as all differences between the four tasks were non-significant [coef $( \pm \mathrm{SE}): 0.05( \pm 0.07) ; t=0.81, p=0.41$ ].

As expected, average $( \pm \mathrm{SD})$ saccade latencies increased from $162 \mathrm{~ms}( \pm 20.9)$ for pure gap to $217 \mathrm{~ms}( \pm 21.3)$ for pure overlap blocks. Mixing gap and overlap rendered the latency for gap trials similar to those in overlap trials; we found only marginally (non-significant) shorter latencies for gap trials $(200 \pm 16.9)$ than for overlap trials $(207 \pm 22.5)$ in mixed blocks, on average. The overall differences between the four tasks were significant [coef $( \pm$ SE): 11.00 ( \pm 1.86$) ; t=5.90, p<0.001$ : see Table 1A], while the post hoc comparisons showed that only the latency in pure gap trials was significantly shorter compared to all other tasks [pure gap vs. pure overlap: coef $( \pm \mathrm{SE}): 45.80( \pm 5.19) ; t=8.83$, $p<0.001$; pure gap vs. mixed gap: coef $( \pm \mathrm{SE}): 13.91( \pm 6.57)$; $t=2.12, p=0.03$; pure gap vs. mixed overlap: coef $( \pm \mathrm{SE}): 0.02$ $( \pm 0.01) ; t=1.76, p=0.12]$.

\section{PUPIL DATA}

Most interestingly, using the gap and overlap tasks showed substantial differences in pupil size (see Figure 2 for average and Figure 3 for individual pupil responses): for pure blocks, the pupil dilation from $200 \mathrm{~ms}$ before target onset to target onset was 0.30 $( \pm 0.2)$ for gap and only $0.07( \pm 0.1)$ for overlap blocks, on average.

Mixing gap and overlap tasks made the pupil dilations more similar, with a slightly (non-significant) larger pupil dilation for gap trials $(0.16 \pm 0.1)$ than for overlap trials $(0.9 \pm 0.2)$, on average. The overall difference in pupil dilations across tasks was significant [coef $( \pm \mathrm{SE}):-0.09( \pm 0.02) ; t=-4.05, p<0.001$ : see Table 1B] but only the difference between the pure gap block and all other blocks was significant in post hoc comparisons [pure gap vs. pure overlap: coef $( \pm \mathrm{SE}):-0.19( \pm 0.7) ; t=-2.99, p=0.002$; pure gap vs. mixed gap: coef $( \pm \mathrm{SE}):-0.10( \pm 0.07) ; t=-1.46$, $p=0.15$; pure gap vs. mixed overlap: coef $( \pm S E):-0.15( \pm 0.08)$; $t=-1.87, p=0.06]$.

Note that, as can be seen in Figure 2, the difference between the different tasks continued to increase $100 \mathrm{~ms}$ after target appearance. We calculated the pupil dilation for the period [-200 100] as well in order to include a broader time period prior to the response which included the knowledge of the target position; strictly speaking, if one were to doubt that there might already be some motor preparation prior to target appearance, this broader time period certainly contained motor preparation as the target location was known. Generally, the pupil dilation ( $z$-scores) was larger for all 
Table 1 | Summary of the mixed-effects models for saccade latency (A) and pupil dilations (B,C).

\begin{tabular}{|c|c|c|c|c|c|c|}
\hline \multicolumn{7}{|c|}{ (A) SACCADE LATENCY (MS) } \\
\hline \multicolumn{5}{|l|}{ Fixed effect } & \multicolumn{2}{|c|}{ Confidence interval (95\%) } \\
\hline Name & Estimate & SE & $t$-Value & $p$-Value & Lower limit & Upper limit \\
\hline (Intercept) & 173.72 & 6.62 & 26.25 & $<0.01$ & 158.75 & 189.65 \\
\hline Tasks & 11.00 & 1.85 & 5.90 & $<0.01$ & 7.34 & 14.66 \\
\hline \multicolumn{7}{|c|}{ Random effect } \\
\hline Group & Name & Variance & SD & & & \\
\hline \multicolumn{7}{|c|}{ (B) PUPIL DILATION [-200 0] (Z-SCORES) } \\
\hline \multicolumn{5}{|l|}{ Fixed effect } & \multicolumn{2}{|c|}{ Confidence interval (95\%) } \\
\hline Name & Estimate & SE & $t$-Value & $p$-Value & Lower limit & Upper limit \\
\hline (Intercept) & 0.35 & 0.06 & 5.48 & $<0.01$ & 0.21 & 0.49 \\
\hline Tasks & -0.09 & 0.02 & -4.05 & $<0.01$ & -0.13 & -0.05 \\
\hline \multicolumn{7}{|c|}{ (C) PUPIL DILATION [-200 100] (Z-SCORES) } \\
\hline \multicolumn{5}{|l|}{ Fixed effect } & \multicolumn{2}{|c|}{ Confidence interval (95\%) } \\
\hline Name & Estimate & SE & $t$-Value & $p$-Value & Lower limit & Upper limit \\
\hline (Intercept) & 0.56 & 0.09 & 5.71 & $<0.01$ & 0.35 & 0.78 \\
\hline Tasks & -0.14 & 0.03 & -4.22 & $<0.01$ & -0.20 & -0.07 \\
\hline \multicolumn{7}{|c|}{ Random effect } \\
\hline Group & Name & Variance & SD & & & \\
\hline Participants & (Intercept) & 0.03 & 0.16 & & & \\
\hline Residuum & & 1.01 & 1.01 & & & \\
\hline
\end{tabular}

$S E$, standard error; $S D$, standard deviation.

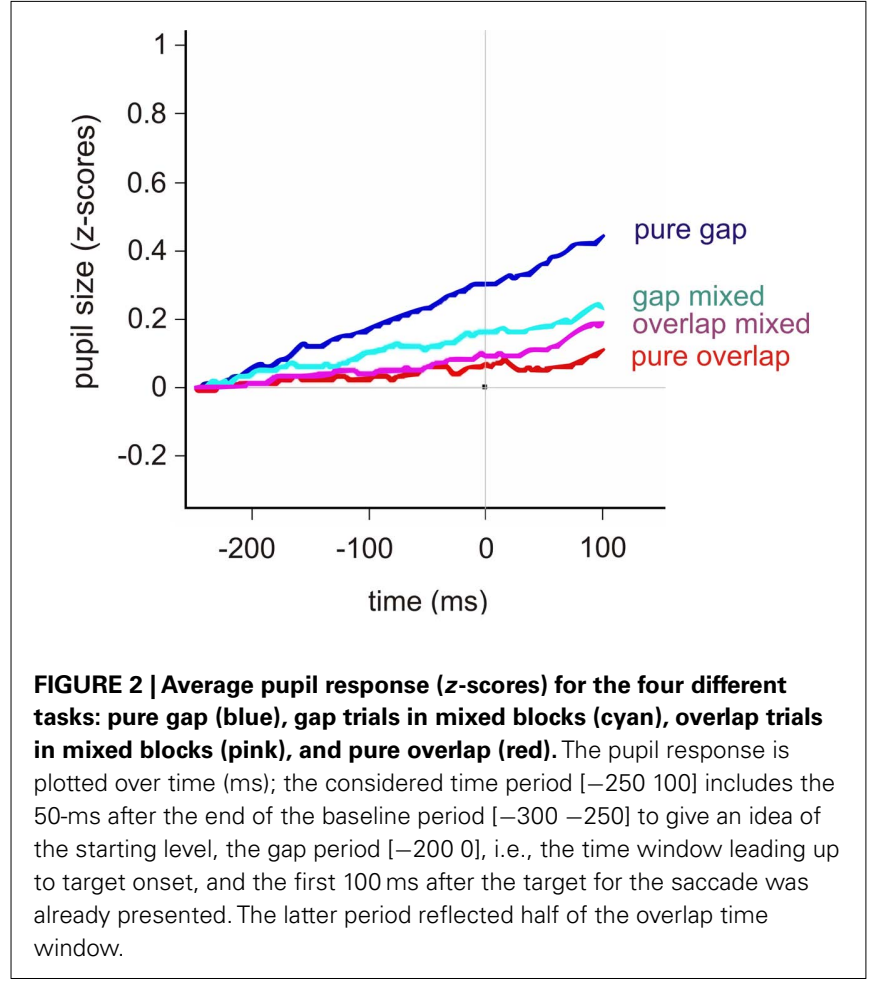

tasks, while for pure blocks, the pupil dilation from $200 \mathrm{~ms}$ before to $100 \mathrm{~ms}$ after target onset was $0.44( \pm 0.3)$ for gap and $0.11( \pm 0.2)$ for overlap blocks, on average; again, mixing made the pupil dilation more similar (gap trials: $0.23 \pm 0.2$; overlap trials: $0.19 \pm 0.2$ ). As for the pupil dilation across the time period [-200 0], the overall differences were significant [coef $( \pm \mathrm{SE}):-0.19( \pm 0.03)$; $t=-4.22, p<0.001$ : see Table 1C] and the post hoc comparisons showed identical results for this broader time window [pure gap vs. pure overlap: coef $( \pm \mathrm{SE}):-0.23( \pm 0.09) ; t=-2.34, p=0.02$; pure gap vs. mixed gap: coef $( \pm \mathrm{SE}):-0.15( \pm 0.12) ; t=-1.13$, $p=0.25$; pure gap vs. mixed overlap: coef $( \pm S E):-0.26( \pm 0.12)$; $t=-2.13, p=0.03$.

\section{COMPARING SACCADE LATENCIES AND PUPIL DILATIONS}

We were mainly interested in the correspondence between pupil dilation and saccade latency and the different saccade tasks were only used to produce a variety of saccade latencies. According to this approach, pupil dilations and analyses were additionally plotted as a function of saccade latency across all tasks (see Figure 4A). This time saccade latency was included into a mixed linear mixed-effects model as a fixed factor, while participants were again treated as a random factor. The corresponding analysis showed that there was a significant decrease in pupil dilation with increasing saccade latency [coef $( \pm \mathrm{SE}):-0.002( \pm 0.0004)$; $t=-5.83, p<0.001$ : see Table 2]. As can be seen additionally in 


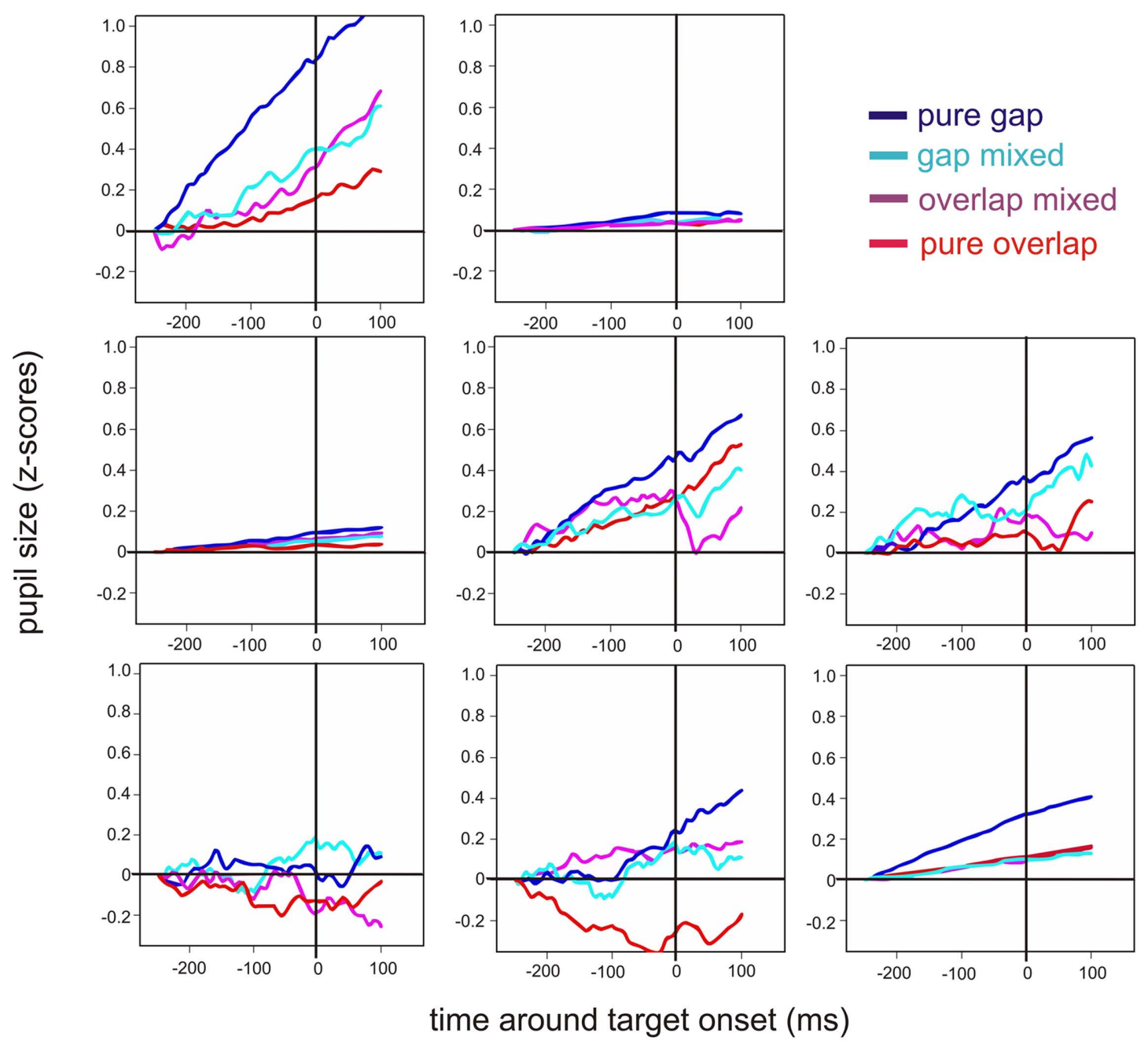

FIGURE 3 | Individual plots for the eight participants for the each pupil response (z-scores): pure gap (blue), gap trials in mixed blocks (cyan), overlap trials in mixed blocks (pink), and pure overlap (red). The pupil response is plotted over time (ms); the considered time period [-250 100] includes the 50-ms after the end of the baseline period [ $-300-250]$ to give an idea of the starting level, the gap period [ -200 0], i.e., a time window up to target onset, and the first $100 \mathrm{~ms}$ after the target for the saccade was already presented. The latter period reflected half of the overlap time window.
Figure 4B, not all participants showed the latter relationship; some participants showed almost no reaction in pupil size changes, as already shown in Figure 3. Furthermore, it was obvious that the range of saccade latencies varied between participants as well.

\section{ARE PUPIL DILATIONS DUE TO CHANGES IN THE FIXATION POINT?}

As mentioned above, during gap trials no fixation target was presented $200 \mathrm{~ms}$ before target appearance while in overlap trials the central fixation point was still presented. Thus, in overlap tasks, higher luminosity could have eventually influenced the pupil size, i.e., induced a decrease in pupil size; therefore, it was important to know if the observed pupil dilation was independent of these physical arangements.

One argument can be leveled against the hypothesis according to which the reported effects were due to this change in stimulus presentation: pupil dilation tended to be smaller for gap trials in mixed blocks compared to pure blocks [coef $( \pm S E):-0.10$
$( \pm 0.07) ; t=-1.46, p=0.15]$. This observation indicates that the change in pupil dilation during the gap period was not only due to the disappearance of the fixation stimulus. Nevertheless, it could be argued that the pupil response during gap trials in mixed blocks (in contrast to pure gap blocks) could have been influenced by the preceding trials; in detail, a carry-over effect could have caused the pupil response to be different for gap trials following an overlap trial compared to gap trials following a gap trial. These objections can be removed in two ways:

(1) The baseline periods of static central fixations before saccades (in pixel) were not statistically different between the four different tasks [pure gap, pure overlap, gap trial in mixed blocks and overlap trials in mixed blocks; coef $( \pm \mathrm{SE}): 0.08( \pm 0.10)$; $t=0.79, p=0.43$ ] - nor were the baseline periods different (a) between pure gap trials and gap trials following a gap trial in mixed blocks [coef $( \pm \mathrm{SE}):-0.06( \pm 0.15) ; t=0.63, p=0.42]$ 


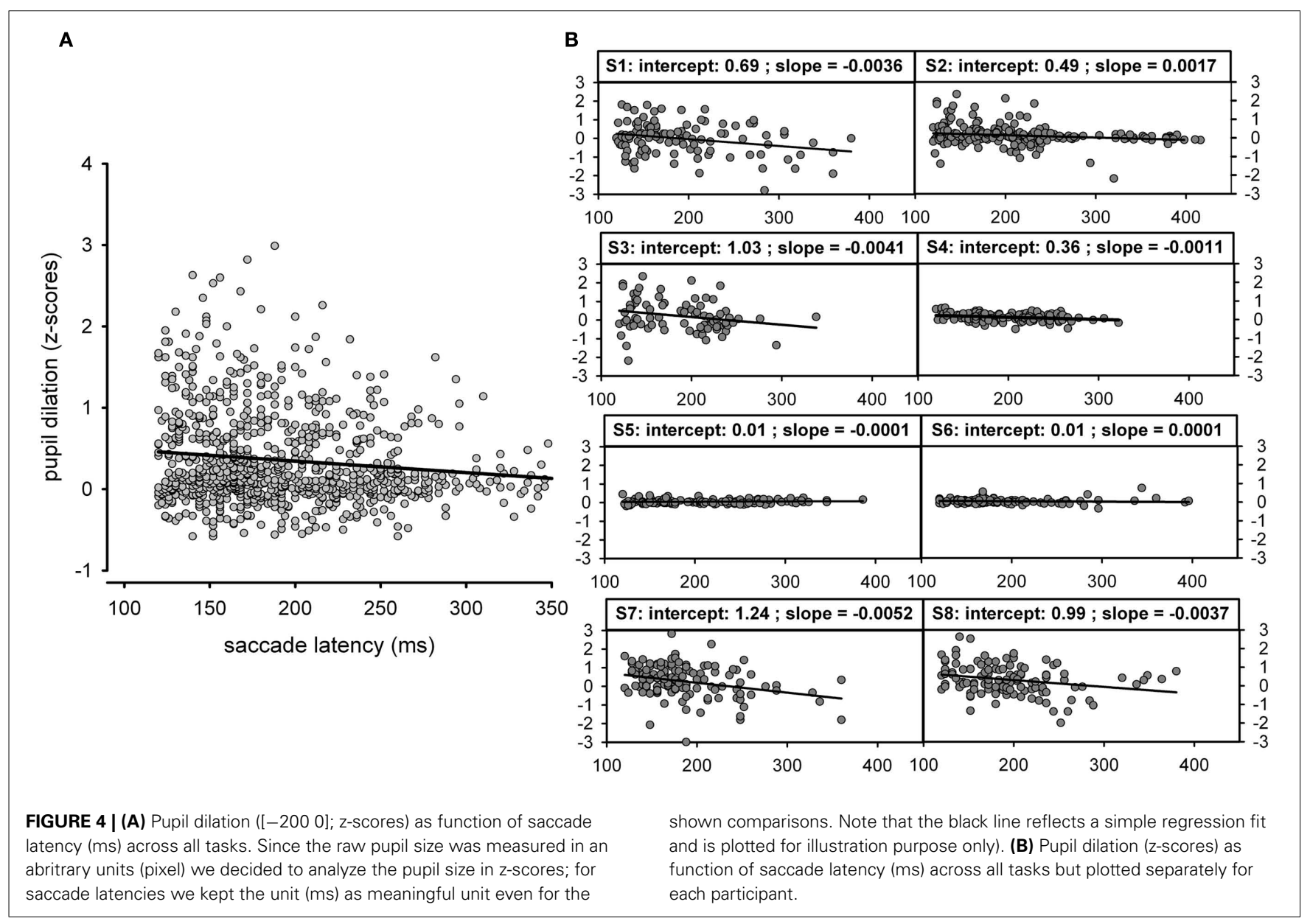

Table 2 | Summary of the mixed-effects model for pupil dilation (z-scores) as a function of saccade latency (ms).

\begin{tabular}{|c|c|c|c|c|c|c|}
\hline Name & Estimate & SE & $t$-Value & $p$-Value & lower limit & upper limit \\
\hline Latency & -0.0022 & 0.0004 & -5.83 & $<0.01$ & -0.0030 & -0.0015 \\
\hline \multicolumn{7}{|c|}{ Random effect } \\
\hline Group & Name & Variance & SD & & & \\
\hline Residuum & & 0.4524 & 0.6726 & & & \\
\hline
\end{tabular}

SE, standard error; SD, standard deviation.

or (b) between pure gap trials and gap trials following an overlap trial in mixed blocks [coef $( \pm \mathrm{SE}): 0.02( \pm 0.09) ; t=0.19$, $p=0.85]$ or (c) gap trials following a gap trial and gap trials following an overlap trial - both, in mixed blocks [coef $( \pm \mathrm{SE})$ : $-0.16( \pm 0.09) ; t=-1.83, p=0.26]$.

(2) We visually inspected the trial sequence in order to observe the time course of the pupil response; if the pupil had still been changing dynamically - because of the previous trial we would have seen an incomplete return to the baseline before the start of a new trial. Such an enduring increase or decrease of the pupil response across the fixation period, i.e., when the eyes once again returned to fixate centrally, was not observed. Figure 5 shows typical pupil responses; we displayed examples of single trials for all four tasks, including the version signal for the eye movement which showed the sequence of a saccade from central fixation to target position and back to central fixation. It could clearly be stated that the pupil response following the target presentation usually ended before the eyes were moved to re-fixate centrally again. 
A
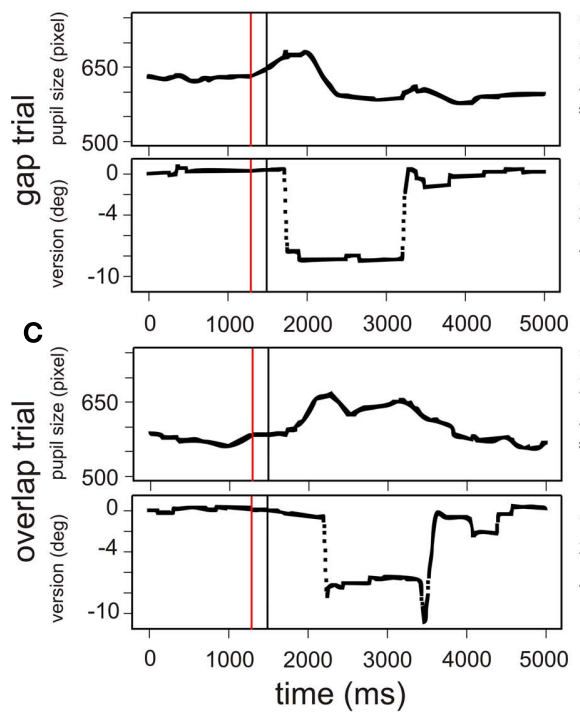

time (ms)
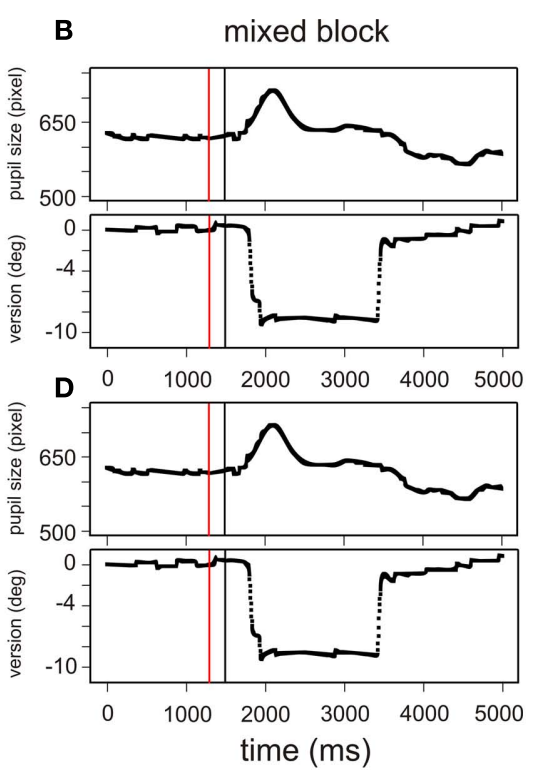

FIGURE 5 | Examples of the typical pupil dilation during the four different tasks: a gap trial in a pure (A) and mixed block (B) and an overlap trial in a pure (C) and mixed block (D), respectively. Pupil data and the corresponding saccadic movements are shown for a single trial (participant 1); the time window [0 5000] corresponds to the sequence "start of central fixation - gap period - target presentation - fixation at target destination - presentation of central target and central fixation." The red line marks the beginning and the black line marks the end of the period [-200 0], for which we analyzed the pupil dilation (for a detailed view on the period [-200 0] see Figure 2).

Accordingly, the black line marks the presentation of the saccade target.

\section{DISCUSSION}

\section{SUMMARY OF THE RESULTS}

The main results are the following. (a) A gap effect (shorter latencies for saccades in gap than in overlap trials) was replicated. Further (b) mixing gap and overlap trials showed switching costs, i.e., saccade latencies increased for gap trials. (c) Pupil dilations prior to saccade execution were larger for gap than for overlap tasks and switching costs were also present in the pupil data. (d) Saccade latencies across different gap and overlap tasks significantly affect pupil dilation prior to the eye movement's execution.

\section{PUPIL DILATIONS IN GAP AND OVERLAP TASKS}

Using video-based eye-tracking devices provides researchers with technologies that sample accurate eye position data and, as shown here, valuable pupil size information. In our case, the standard gap/overlap paradigm induced expected effects in saccade latencies, i.e., it produced different kinds of motor preparation. Most interestingly, the pupil dilation resembled the pattern of saccade latencies: the pupil dilated more in gap compared to overlap blocks. Thus, pupil dilation clearly reflected task variations, as described before (Kahneman et al., 1969; Hess and Howell, 1988; Beatty and Brennis, 2000; Barbur, 2004; Porter et al., 2007; Conway et al., 2008). Moreover, we found a significant effect of saccade latencies on pupil dilations across all tasks. In other words, knowing saccade latencies allowed for predicting - post hoc - pupil dilations. Note that all of our pupil data were extracted from fixation periods during which the eyes did not move - that is from a period of $200 \mathrm{~ms}$ prior to target onset, i.e., about $300-400 \mathrm{~ms}$ before actual saccade execution. Even if there were short reflexive movements of the pupil between trials, the statistics confirmed that the baselines of pupil sizes were not different between different tasks. Moreover, theoretically, in overlap tasks, higher luminosity might have influenced the pupil size, because in gap tasks there was nothing on the screen while in overlap tasks the fixation point was presented (see Figure 1). Regarding this slight luminance change during gap trials (i.e., when the central dot was switched off) the change in pupil dilation during this gap period was not only due to this disappearance of the fixation stimulus. Had such been the case, the pupil dilation would have been about the same for all gap trials - which was not the case. Additionally, across all observations a linear relationship between pupil dilation and saccade latency could be found, which could not be produced by a pure luminance change between gap and overlap trials. Thus, average pupil dilations were more likely to be correlated with saccade latencies produced by different saccadic tasks.

In keeping with Hupe et al. (2009), the pupil dilation in our data was also initiated by an event that happened as early as about $300 \mathrm{~ms}$ before the actual saccade execution. Responselocked data for speeded reactions also suggested very early reflection of response preparations within the pupil response (Richer et al., 1983; Richer and Beatty, 1985). Regarding the explanations about the underlying processes of the effects in saccade latency due to gap and overlap variations in pure and mixed blocks (Fischer et al., 1993; Kingstone and Klein, 1993; Klein et al., 1995; Rolfs and Vitu, 2007; Vernet et al., 2009), we would conclude that the found effect in pupil dilations reflected differences in motor preparations: the shorter the latencies and the larger the pupil dilation, the more advanced the motor preparation. It therefore follows that our present results are congruent with data Karatekin et al. (2010) reported for the comparison of pro- and anti-saccades: 
the anti-saccade task produced longer latencies, when compared to the pro-saccade task, and in parallel, absolute pupil diameters increased for anti-saccades. Moreover, the moment in time after target onset at which the pupil diameter peaked was delayed in the anti-saccade task. Unfortunately, this reported pupil response data was aligned to target onset and thus, all variation in pupil diameter prior to target onset could not be retrieved from the report. We were also confronted with questions concerning how possible artifacts in the pupil diameter recordings were eliminated; as can easily be seen in Figure 5 the rapid movement of the eye and the change in the observation angle directly affects pupil size measures when using a video-based technique. In order to avoid these possible confounding artifacts, we concentrated the present study, as mentioned above, on pupil responses prior to saccade movements, i.e., while the eye was steadily fixating the central fixation dot. Regardless of the aforementioned methodological issues, both studies, the one conducted by Karatekin et al. (2010) and our present study, suggest that pupil dilation accompanies motor preparation; given that actual motor preparation for eye movements away from the target is delayed in anti-saccades tasks (because reflexive preparations toward the target have to be inhibited first). And indeed, the previously reported delayed pupil dilation also supported this idea.

Nevertheless, alternative explanations for pupil responses prior to movement executions or even prior to target appearance are also discussed in the literature. For example, Jennings et al. (1998) measured several psychophysiological parameters, including pupil size while they varied the foreperiod of a reaction time task. They showed that pupil dilations were larger prior to stimulus appearance during longer foreperiods; moreover, while using a non-aging foreperiod manipulation, the temporal expectations of reaction stimulus were reduced as were pupil dilations in parallel. However, they failed to show a correlation between momentary pupil dilations and actual reaction times, since all reported pupil size changes emerged as slow, tonic changes, while the non-aging foreperiod manipulation equalized reaction times across different foreperiods. Based on these results, the authors concluded that

\section{REFERENCES}

Baayen, R. H., Davidson, D. J., and Bates, D. M. (2008). Mixed-effects modeling with crossed random effects for subjects and items. J. Mem. Lang. 59, 390-412.

Barbur, J. L. (2004). "Learning from the pupil-studies of basic mechanisms and clinical application," in The Visual Neurosciences, Vol. 1, eds L. M. Chalupa and J. S. Werner (Cambridge: MIT Press), 641-656.

Beatty, J., and Brennis, J. W. (2000). “The pupillary system," in Handbook of Psychophysiology, eds J. T. Cacioppo, G. Berntson, and L. G. Tassinary (Cambridge: Cambridge University Press), 142-162.

Breen, 1. A., Burde, R. M., and Loewy, A. D. (1983). Brainstem connections to the Edinger-Westphal nucleus of the cat: a retrograde tracer study. Brain Res. 261, 303-306.
Conway, C. A., Jones, B. C., DeBruine, L. M., Little, A. C., and Sahraie, A. (2008). Transient pupil constrictions to faces are sensitive to orientation and species. J. Vis. 8, 1-11.

Dorris, M. C., Pare, M., and Munoz, D. P. (1997). Neuronal activity in monkey superior colliculus related to the initiation of saccadic eye movements. J. Neurosci. 17, 8566-8579.

Findlay, J. M., and Walker, R. (1999). A model of saccade generation based on parallel processing and competitive inhibition. Behav. Brain Sci. 22, 661-674; discussion 674-721.

Fischer, B., Weber, A., Biscaldi, M., Aiple, F., Otto, P., and Stuhr, V. (1993). Separate populations of visually guided saccades in humans: reaction times and amplitudes. Exp. Brain Res. 92, 528-541.

their finding should be interpreted in favor of the concept of preparation which includes a transient organization of different processes, while each of these processes are only modestly related to the actual response. In contradistinction from these findings our results point toward a direct relation between reaction times, i.e., saccade latencies, and pupil dilations and thus, we interpret, as stated above, this observed relation as support for the hypothesis of specific preparation as a potential indicator of motor preparation.

The eye provides a unique linkage between different branches of the body: the eye muscles direct the eyes to different targets, while the sphincter and dilator muscle of the iris (in tandem with the ciliar muscle, which regulates accommodation separately) optimize the optical image qualities of the perceived target; that is, more or less cortically controlled mechanisms work in the close neighborhood of systems directly influenced via sympathetic and parasympathetic inputs. As has been shown for the pupil light reflex, the parasympathetic pathway can be (1) modulated at the level of the pretectal olivary nucleus, which receives multiple cortical and sub-cortical inputs (Barbur, 2004; Gamlin, 2006) or (2) influenced by the Edinger-Westphal nucleus, which receives inhibitory influences in particular from the locus coeruleus, the cortex and the ascending reticular system (Loewenfeld, 1958, 1999; Breen et al., 1983; Merritt et al., 2004); further, for the sympathetic pathway, the integration of all activity (including cortical activation via thalamic/hypothalamic areas) is brought together in the posterior hypothalamus as the beginning of the final source of direct sympathetic stimulation of the pupillary dilator. From one point of view, it is not surprising that an advanced motor preparation is mirrored by corresponding pupil dilations; if the eye is prepared to move, a (perhaps residual) projection into areas providing a modulation in the autonomous pathways (in terms of a reduced parasympathetic and/or increased sympathetic influence) might be a possible consequence.

\section{ACKNOWLEDGMENTS}

The authors wish to thank Gabi Lipede for his help in checking the final version of the manuscript for language errors.

Gamlin, P. D. (2006). The pretectum: connections and oculomotorrelated roles. Prog. Brain Res. 151, 379-405.

Goldring, J., and Fischer, B. (1997). Reaction times of vertical prosaccades and antisaccades in gap and overlap tasks. Exp. Brain Res. 113, 88-103.

Hess, R. F., and Howell, E. R. (1988). Detection of low spatial frequencies: a single filter or multiple filters? Ophthalmic Physiol. Opt. 8, 378-385.

Hupe, J.-M., Lamirel, C., and Lorenceau, J. (2009). Pupil dynamics during bistable motion perception. J. Vis. $9,10$.

Isa, T., and Kobayashi, Y. (2004). Switching between cortical and subcortical sensorimotor pathways. Prog. Brain Res. 143, 299-305.

Jennings, J. R., van der Molen, M. W., and Steinhauer, S. R. (1998).
Preparing the heart, eye, and brain: foreperiod length effects in a nonaging paradigm. Psychophysiology 35, 90-98.

Kahneman, D., Tursky, B., Shapiro, D., and Crider, A. (1969). Pupillary, heart rate, and skin resistance changes during a mental task. J. Exp. Psychol. 79, 164-167.

Karatekin, C., Bingham, C., and White, T. (2010). Oculomotor and pupillometric indices of proand antisaccade performance in youth-onset psychosis and attention deficit/hyperactivity disorder. Schizophr. Bull. 36, 1167-1186.

Kingstone, A., and Klein, R. M. (1993). Visual offsets facilitate saccadic latency: does predisengagement of visuospatial attention mediate this gap effect? J. Exp. Psychol. Hum. Percept. Perform. 19, 1251-1265. 
Klein, R. M., Taylor, T. L., and Kingstone, A. (1995). Against a role for attentional disengagement in the gap effect: a friendly amendment to Tam and Stelmach (1993). Percept. Psychophys. 57, 573-577.

Loewenfeld, I. E. (1958). Mechanisms of reflex dilatation of the pupil; historical review and experimental analysis. Doc. Ophthalmol. Proc. Ser. 12, 185-448.

Loewenfeld, I. E. (1999). The Pupil: Anatomy, Physiology, and Clinical Applications. Boston: ButterworthHeinemann.

Lowenstein, O., and Loewenstein, I. E. (1969). "The pupil," Chapter 9, in The Eye, ed. H. Dovson (New York: Academic Press), 13-337.

Merritt, S. L., Schnyders, H. C., Patel, M., Basner, R. C., and O’Neil, W. (2004). Pupil staging and EEG measurement of sleepiness. Int. J. Psychophysiol. 52, 97-112.

Munoz, D. P., and Wurtz, R. H. (1992). Role of the rostal superior colliculus in active visual fixation and execution of express saccades. J. Neurophysiol. 67, 1000-1002.

Pinheiro, J. C., and Bates, D. M. (2000). Mixed-Effects Models in S and S-Plus. New York: Springer.
Porter, G., Troscianko, T., and Gilchrist, I. D. (2007). Effort during visual search and counting: insights from pupillometry. Q. J. Exp. Psychol. 60, 211-229.

R-Development-Core-Team. (2008). $R$ : A Language and Environment for Statistical Computing. Available at: www.r-project.org

Richer, F., and Beatty, J. (1985). Pupillary dilations in movement preparation and execution. Psychophysiology 22, 204-207.

Richer, F., Silverman, C., and Beatty, J. (1983). Response selection and initiation in speeded reactions: a pupillometric analysis. J. Exp. Psychol. Hum. Percept. Perform. 9, 360-370.

Rolfs, M., and Vitu, F. (2007). On the limited role of target onset in the gap task: support for the motorpreparation hypothesis. J. Vis. 7, $1-20$.

Ross, J., Burr, D., and Morrone, C. (1996). Suppression of the magnocellular pathway during saccades. Behav. Brain Res. 80, 1-8.

Saslow, M. G. (1967). Effects of components of displacement-step stimuli upon latency for saccadic eye movement. J. Opt. Soc. Am. 57, 1024-1029.
Schiller, P. H., Sandell, J. H., and Maunsell, J. H. (1987). The effect of frontal eye field and superior colliculus lesions on saccadic latencies in the rhesus monkey. J. Neurophysiol. 57, 1033-1049.

Schiller, P. H., and Tehovnik, E. J. (2005). Neural mechanisms underlying target selection with saccadic eye movements. Prog. Brain Res. 149 157-171.

Venables, W. N., and Smith, D. M (2001). An Introdcution to R. Available at: www.r-project.org

Vernet, M., Yang, Q., Gruselle, M., Trams, M., and Kapoula, Z. (2009). Switching between gap and overlap pro-saccades: cost or benefit? Exp. Brain Res. 197, 49-58.

West, B. T., Welch, K. B., and Gallechki, A. T. (2007). Linear Mixed Models. A Practical Guide Using Statistical Software. Boca Raton: Chapman and Hall.

Wilhelm, B. J., Wilhelm, H., Moro, S., and Barbur, J. L. (2002). Pupil response components: studies in patients with Parinaud's syndrome. Brain 125, 2296-2307.

Yang, Q., Bucci, M. P., and Kapoula, Z. (2002). The latency of saccades, vergence, and combined eye movements in children and in adults Invest. Ophthalmol. Vis. Sci. 43, 2939-2949.

Conflict of Interest Statement: The authors declare that the research was conducted in the absence of any commercial or financial relationships that could be construed as a potential conflict of interest.

Received: 26 May 2011; accepted: 19 August 2011; published online: 30 September 2011.

Citation: Jainta $S$, Vernet $M$, Yang $Q$ and Kapoula $Z$ (2011) The pupil reflects motor preparation for saccades - even before the eye starts to move. Front. Hum. Neurosci. 5:97. doi: 10.3389/fnhum.2011.00097

Copyright (c) 2011 Jainta, Vernet, Yang and Kapoula. This is an open-access article subject to a non-exclusive license between the authors and Frontiers Media $S A$, which permits use, distribution and reproduction in other forums, provided the original authors and source are credited and other Frontiers conditions are complied with. 


\section{APPENDIX}

For the presented pupil data the distance between eye and camera was not available - as well as calibrated pupil diameter in millimeters. We therefore decided to measure the distance between right eye and Eyelink II camera post hoc for a new sample of 10 participants who took part in another experiment which was run within a set-up comparable to the one described above. As can be seen in Figure A1 the actual distance between eye and camera ranged between 5.3 and $6.7 \mathrm{~cm}$.

Additionally, after the camera was adjusted properly, eye movement data collected and the participants had been given a break within a sequence of runs, we fixed a white piece of paper with black dots of 4 and $6 \mathrm{~mm}$ in diameter at the same distance as the eye prior has been. We then collected short streams of data (about $500 \mathrm{~ms}$ ) for each dot, simulating pupil diameters. For each diameter we randomly sampled up to 10 measures out of every recording and pooled them for each participant's camera adjustment. Analog to the analysis above, we $z$-standardized this data for each participant (i.e., camera distance) and then calculated the difference between the 4- and 6-mm dot presentation. As can be seen in Figure A2 the resulting $z$-scores ranged between 0.53 and 1.08 for a corresponding $2 \mathrm{~mm}$ change in pupil size. Comparing this result with the observed changes described above suggested that most changes we observed were clearly smaller than $2 \mathrm{~mm}-$ most of the time presumably less then $1 \mathrm{~mm}$. According to the Eyelink II manual the system is supposed to resolve $1 \%$ of pupil diameter as standard resolution for pupil size changes that would be $0.04 \mathrm{~mm}$ for the 4-mm dot in our simulated pupil data. Calibrating the raw pixel data of our simulation by using both diameters (i.e., 4 and $6 \mathrm{~mm}$ ) in a linear regression - first, individually and then pooling the data again across trials and participants - gave, on average a change of about 32 pixel $( \pm 9)$ for a pupil size change of $0.04 \mathrm{~mm}$. Transferred into $z$-scores - again, first, calculated individually and then pooled across trials and participants - we see that a change of $0.0198( \pm 0.0002)$ within the $z$-score data corresponded to a $0.04-\mathrm{mm}$ pupil size change, on average.

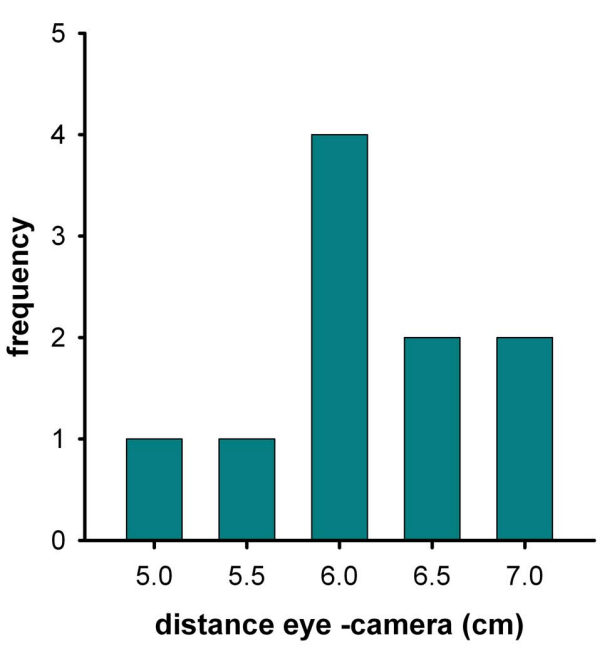

FIGURE A1 | Histogram of the measured distance between eye and Eyelink II camera (in $\mathrm{cm}$ ) for 10 participants.

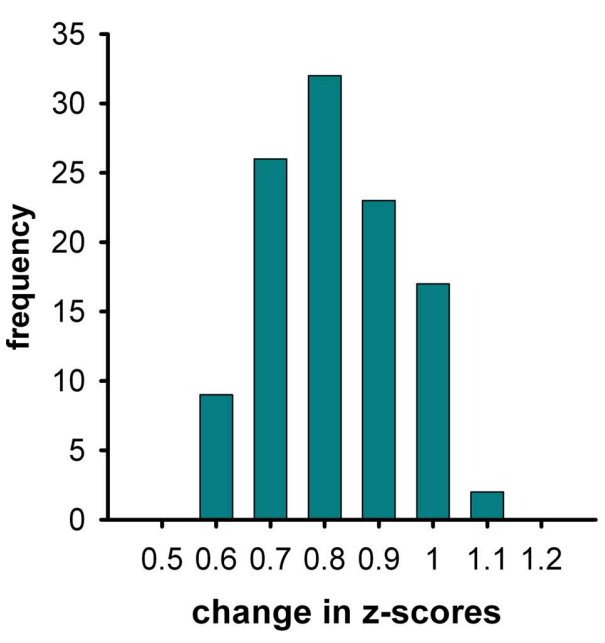

FIGURE A2 | Histogram of the z-scores corresponding to $2 \mathbf{m m}$ simulated change in pupil size. 